\title{
November 2013 Imaging Case of the Month
}

\author{
Michael B. Gotway, MD \\ Department of Radiology \\ Mayo Clinic Arizona \\ Scottsdale, AZ
}

\section{Clinical History}

A 67-year-old man complained of intermittent shortness of breath. A few crackles were audible in the patient's lung bases bilaterally. Chest radiography (Figure 1) was performed.

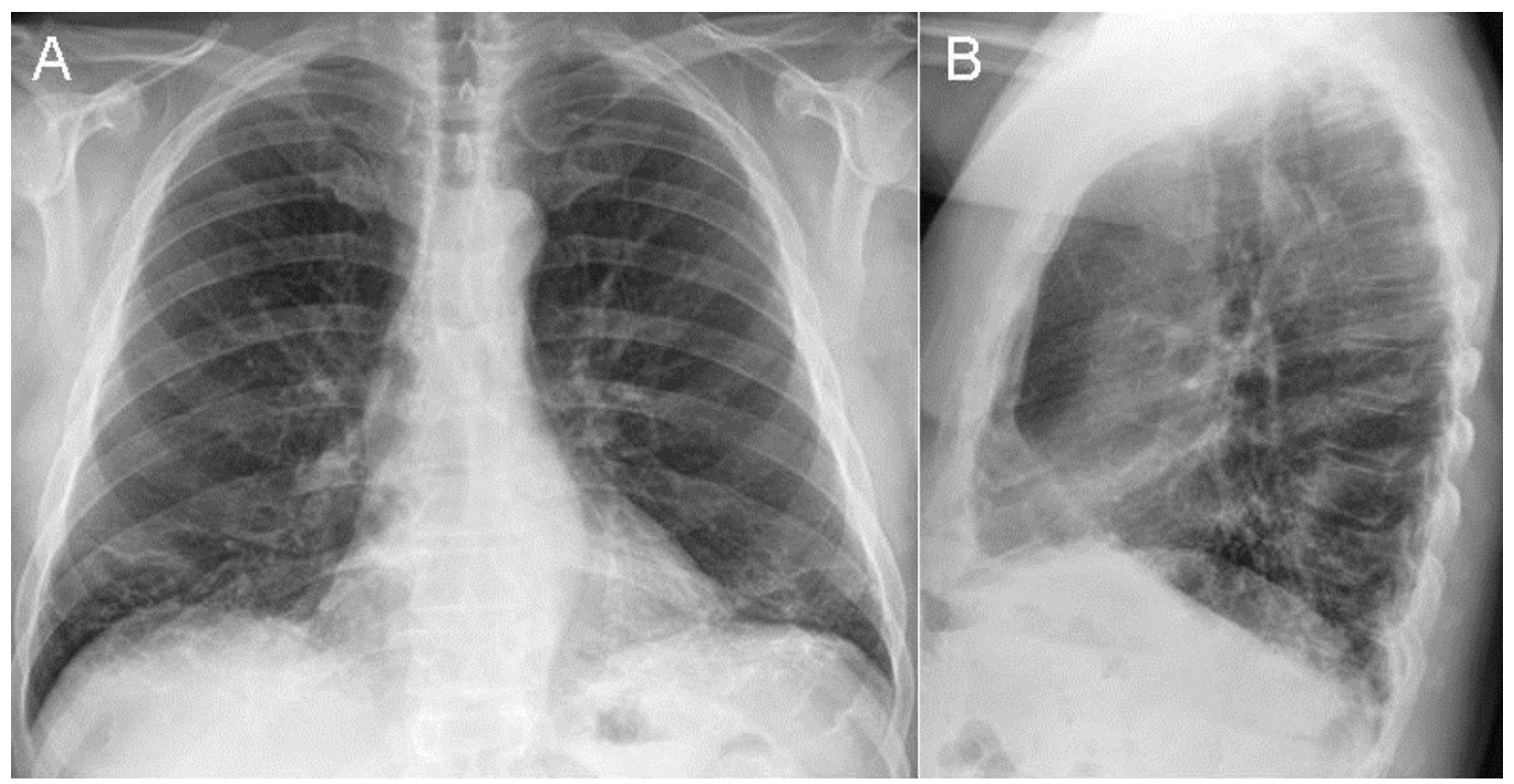

Figure 1: Frontal $(A)$ and lateral $(B)$ chest radiography.

Which of the following statements regarding the chest radiograph is most accurate?

1. The chest radiograph shows basal predominant linear opacities suggesting fibrosis

2. The chest radiograph shows large lung volumes with cystic change

3. The chest radiograph shows multifocal ground-glass opacity and cavitary consolidation

4. The chest radiograph shows multifocal ground-glass opacity and consolidation associated with linear and reticular abnormalities

5. The chest radiograph shows multiple nodules 


\section{Correct! \\ 1. The chest radiograph shows basal predominant linear opacities suggesting fibrosis}

The chest radiograph does not show areas of confluent increased attenuation, such as ground-glass opacity or consolidation. No discrete areas of cavitation are present and no distinct nodules are seen. The chest radiograph does show bilateral, symmetric, predominantly linear and reticular opacities which may suggest fibrotic lung disease, although there is no discrete honeycombing and the lung volumes are not abnormally diminished.

Which of the following is the most appropriate consideration among the differential diagnostic possibilities for the appearance of the patient's chest radiograph?

1. Coccidioidomycosis

2. Granulomatosis with polyangiitis (Wegener's granulomatosis)

3. Increased pressure edema

4. Sarcoidosis

5. Usual interstitial pneumonia / idiopathic pulmonary fibrosis 


\section{Correct! \\ 5. Usual interstitial pneumonia / idiopathic pulmonary fibrosis}

Among the choices listed, the most likely cause for the findings on the chest radiograph is usual interstitial pneumonia / idiopathic pulmonary fibrosis (UIP / IPF). The chest radiographic findings are potentially consistent with this disorder, though certainly not diagnostic of it- the lung volumes are not abnormally decreased, as is often the case with UIP / IPF, nor is there visible honeycombing. Furthermore, the pulmonary opacities in UIP / IPF are often more peripherally distributed, and small reticular opacities in the subpleural lung, rather than the longer linear opacities evident on this image, are commonly seen with UIP / IPF. Linear and reticular opacities with architectural distortion, suggesting fibrotic lung disease, may occur with sarcoidosis, but typically such findings are upper lobe predominant, not lower lobe predominant. Granulomatosis with polyangiitis (Wegener's granulomatosis) often manifests at chest radiography with multiple, bilateral, variably-sized nodules and cavities, or perhaps diffuse pulmonary opacities resulting from pulmonary hemorrhage- neither are evident in this case. The imaging manifestations of coccidioidomycosis are protean, typically including consolidation with lymph node enlargement, miliary disease, single or multiple nodules, or single or multiple cavities of variable wall thickness, among other possibilities; however, basal linear and reticular opacities are typically not a manifestation of pulmonary coccidioidomycosis infection. Finally, basal predominant linear opacities (often representing interlobular septal thickening) are commonly seen in patients with increased pressure edema (congestive heart failure), but usually other radiographic abnormalities are present, typically cardiomegaly, a widened vascular pedicle, and pleural effusions. Such abnormalities are not present in this case.

The patient was treated with broad spectrum antibiotics and diuresis, but was lost to follow up. Four years later, a repeat chest radiograph (Figure 2) was performed when the patient again presented with complaints of intermittent shortness of breath. 


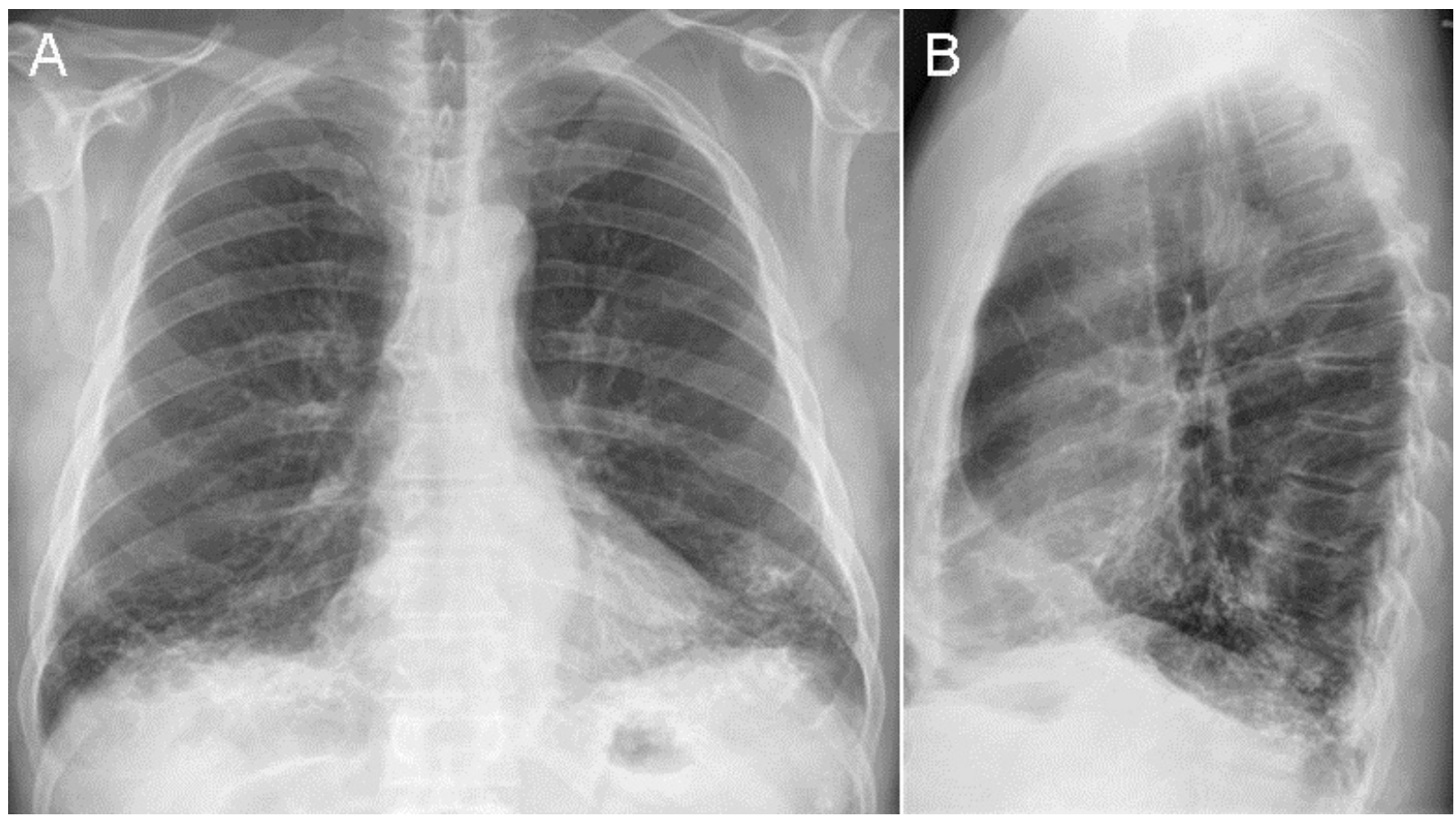

Figure 2. Frontal (A) and lateral (B) chest radiography performed 4 years following Figure 1.

Which of the following statements regarding the chest radiograph is most accurate?

1. The chest radiograph shows new air trapping

2. The chest radiograph shows new pulmonary nodules

3. The chest radiograph shows newly diminished lung volumes compared to the previous study

4. The chest radiograph shows that the findings present previously have resolved

5. The chest radiographic findings present previously have progressed slightly 


\section{Correct!}

\section{The chest radiographic findings present previously have progressed slightly}

The chest radiograph again shows basal predominant linear and reticular abnormalities, suggesting the possibility of fibrotic lung disease. The opacities appear slightly more pronounced compared with prior, but the lung volumes remain normal. No nodules are present. No areas of localized lucency or volume increase to suggest air trapping is seen.

The patient underwent thoracic CT (Figure 3) for further characterization of the abnormalities seen at chest radiography.

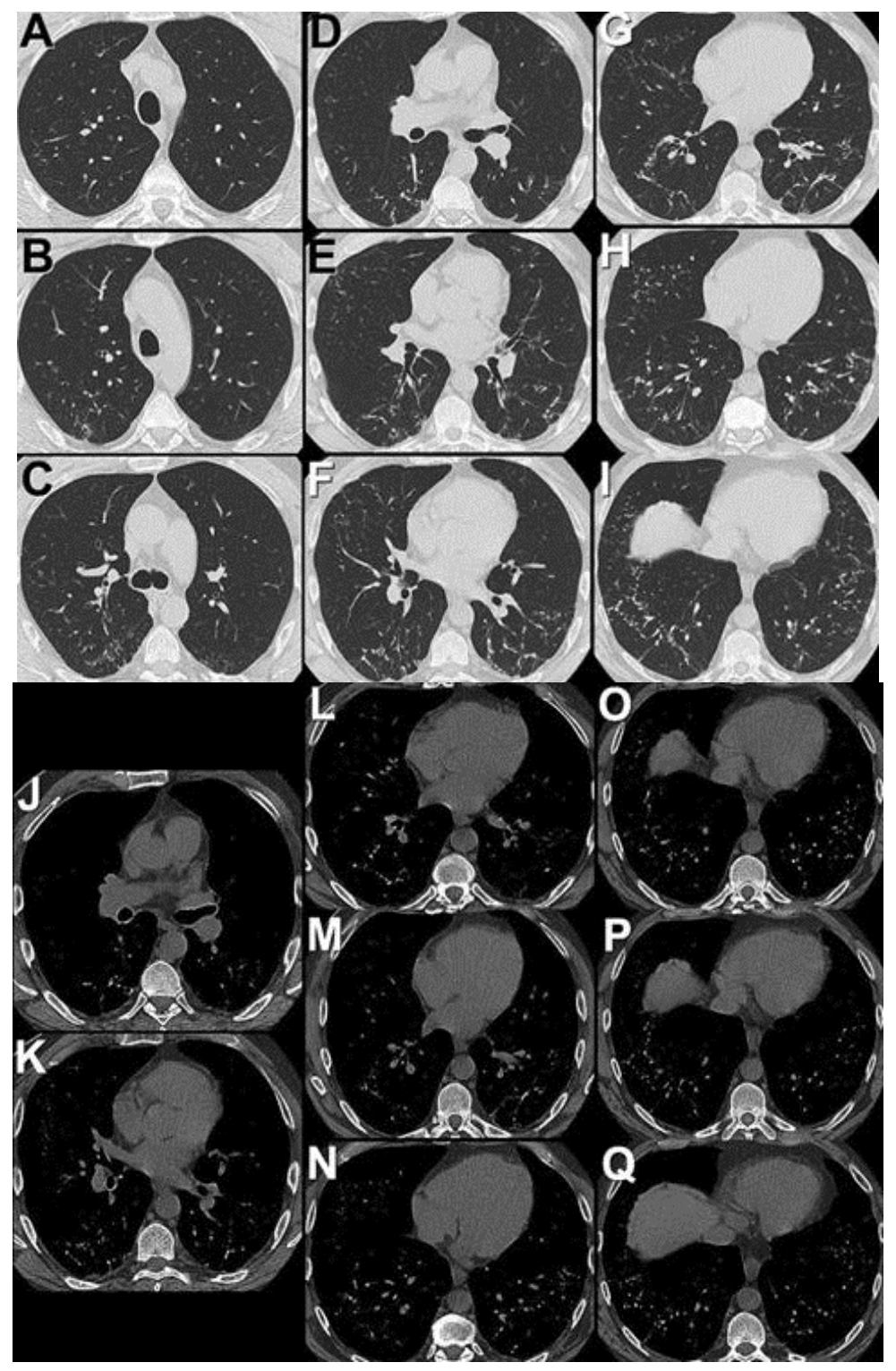

Figure 3. Axial thoracic CT displayed in lung windows (A-I) and soft tissue windows (JQ). 
Which of the following statements regarding this CT examination is most accurate?

1. The thoracic CT shows basal linear and hyperattenuating nodular interstitial thickening

2. The thoracic CT shows basal predominant intralobular interstitial thickening and honeycombing, suggesting fibrosis and consistent with UIP / IPF

3. The thoracic CT shows miliary nodules

4. The thoracic CT shows multifocal bronchiectasis

5. The thoracic CT shows multiple small nodules with "tree-in-bud" morphology 


\section{Correct! \\ 1. The thoracic CT shows basal linear and hyperattenuating nodular interstitial thickening}

Thoracic CT shows basal predominant linear opacities associated with small, circumscribed nodules; the wide window width images (Figure 3J-Q) show that some of the nodular opacities are hyperattenuating, suggesting calcification or ossification. The opacities are not predominantly distributed in the subpleural regions of lung, nor is there honeycombing or intralobular interstitial thickening; the lack of these findings and the lack of the clear, basal and subpleural predominance of the findings argues against the diagnosis of UIP / IPF. While nodules are present, they are distributed in a patchy, peribronchovascular and interstitial manner, not the random pattern typical of a miliary distribution. The nodules do not show the branching morphology characteristic of "treein-bud" opacities. Bronchiectasis is not evident.

What is the appropriate next step for the evaluation / management of this patient?

1. ${ }^{18}$ FDG-PET scanning

2. Bronchoscopy with transbronchial biopsy

3. Open surgical lung biopsy

4. Percutaneous transthoracic needle biopsy

5. Serial imaging to assess for change 


\section{Correct! \\ 2. Bronchoscopy with transbronchial biopsy}

Bronchoscopy with transbronchial biopsy is the most appropriate next step among the choices listed. A case could be made for choice 3. Open surgical lung biopsy. However, video-assisted thoracoscopic surgical biopsy is generally favored over the open form of the procedure for the diagnosis of interstitial lung abnormalities. Furthermore, a surgical lung biopsy may not be required at all if the less morbid and less expensive bronchcoscopic biopsy establishes a diagnosis. Serial imaging to assess for change has little relevance here given that the abnormalities have persisted- indeed, slightly progressed- over time. The small interstitial nodular opacities on this patient's CT are too small to target with percutaneous transthoracic fine needle biopsy. Finally, ${ }^{18}$ FDGPET scan would not be management-altering in this case. First, given the slow progression of the abnormalities, ${ }^{18}$ FDG-PET would likely show no significant tracer accumulation. However, even if tracer accumulation was seen within the pulmonary opacities at ${ }^{18}$ FDG-PET, that finding would be non-specific and a tissue diagnosis would be pursued regardless.

The patient underwent bronchoscopy with transbronchial biopsy. The histopathological material obtained from biopsy is shown in Figure 4.

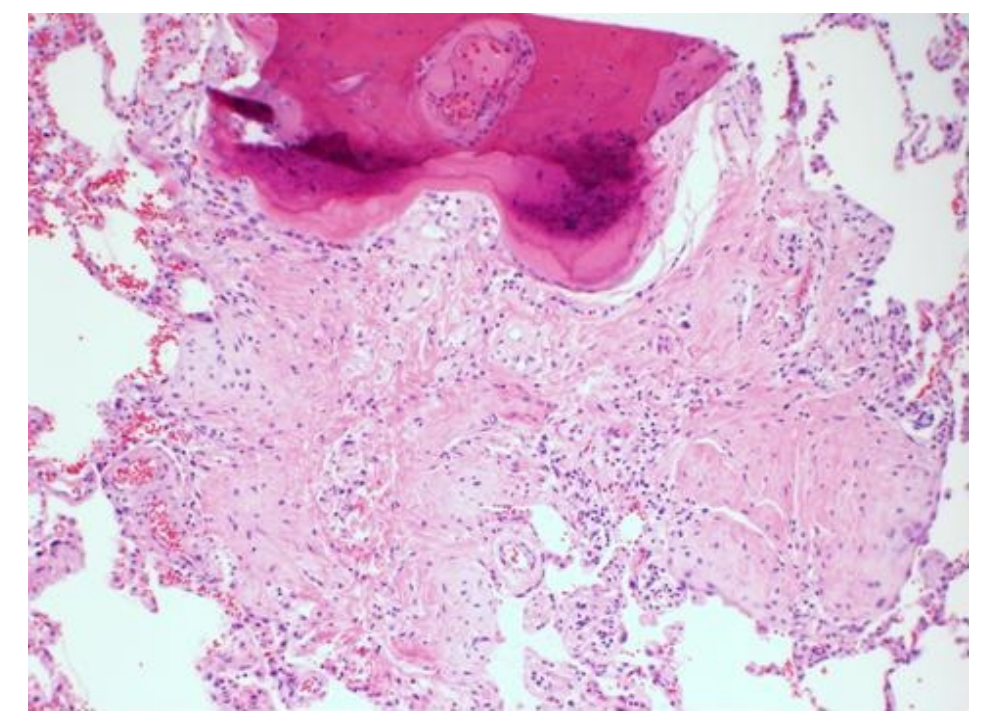

Figure 4. Histopathological specimen obtained at bronchoscopy with transbronchial biopsy

What is the most likely diagnosis?

1. Dendriform pulmonary ossification

2. Non-specific interstitial pneumonia

3. Pulmonary lymphangitic carcinomatosis

4. Sarcoidosis

5. Usual interstitial pneumonia / idiopathic pulmonary fibrosis 


\section{Correct! \\ 1. Dendriform pulmonary ossification}

No fibroblastic foci were identified. Rather nodular and branching foci of metaplastic bone, occasionally protruding into the alveoli, were seen in the biopsy specimen. The pathogenesis of idiopathic pulmonary ossification is unknown. Serum calcium and phosphorus levels are usually normal. Corticosteroids, calcium-binding drugs, and lowcalcium diets have no discernable benefit, although they have not been systematically evaluated; the role of bisphosphonates remains to be determined. Because there is no proven treatment for pulmonary ossification, any therapy directed at the ossification should be considered experimental and reserved only for symptomatic cases.

Diagnosis: Dendriform pulmonary ossification

\section{References}

1. Chan ED, Morales DV, Welsh CH, McDermott MT, Schwarz MI. Calcium deposition with or without bone formation in the lung. Am J Respir Crit Care Med 2002; 165(12):1654-69. [CrossRef] [PubMed]

2. Ndimbie OK, Williams CR, Lee MW. Dendriform pulmonary ossification. Arch Pathol Lab Med 1987;111(11):1062-64. [PubMed]

3. Martinez JB, Ramos SG. Dendriform pulmonary ossification. Lancet 2013 [DOI: S0140-6736(13)60097-5] [CrossRef]

4. Reddy $T L$, von der Thüsen J, Walsh SL. Idiopathic dendriform pulmonary ossification. J Thorac Imaging 2012; 27(5):W108-110. [CrossRef] [PubMed] 